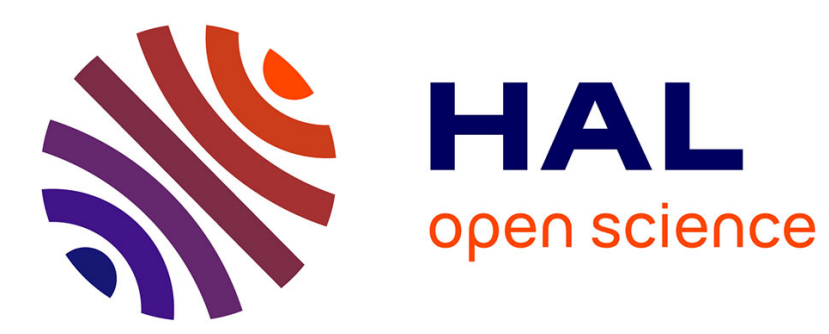

\title{
An adaptive model reduction strategy for post-buckling analysis of stiffened structures
}

Ludovic Barrière, Steven Marguet, Bruno Castanié, Philippe Cresta, Jean-Charles Passieux

\section{- To cite this version:}

Ludovic Barrière, Steven Marguet, Bruno Castanié, Philippe Cresta, Jean-Charles Passieux. An adaptive model reduction strategy for post-buckling analysis of stiffened structures. Thin-Walled Structures, 2013, 73, pp.81-93. 10.1016/j.tws.2013.07.009 . hal-00870773

\section{HAL Id: hal-00870773 https://hal.science/hal-00870773}

Submitted on 8 Oct 2013

HAL is a multi-disciplinary open access archive for the deposit and dissemination of scientific research documents, whether they are published or not. The documents may come from teaching and research institutions in France or abroad, or from public or private research centers.
L'archive ouverte pluridisciplinaire HAL, est destinée au dépôt et à la diffusion de documents scientifiques de niveau recherche, publiés ou non, émanant des établissements d'enseignement et de recherche français ou étrangers, des laboratoires publics ou privés. 


\title{
An adaptive model reduction strategy for post-buckling analysis of stiffened structures
}

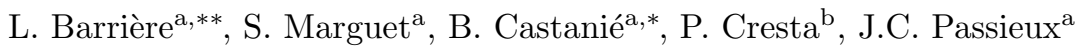 \\ ${ }^{a}$ Institut Clément Ader, 135 av. de Rangueil, 31077 Toulouse, France \\ ${ }^{b}$ EADS Innovation Works, Campus Engineering, CTO IW SP SC, BP 90112, 31703 BLAGNAC Cedex, FRANCE
}

\begin{abstract}
The finite element simulation of structures subjected to post-buckling still faces computational limits, especially for large stiffened structures. Several solving strategies have already been proposed in response to this issue. Among them are the adaptive model reduction solving techniques which demonstrated their ability to drastically reduce the number of unknowns as well as to control the approximation error of solving non-linear problems like post-buckling. The challenges regarding these techniques are the computation of a reduced basis at lower cost, the use of an efficient adaptive procedure and the limitation of the number of call to the adaptive procedure. This paper proposes a Post-Buckling Adaptive Model Reduction (PBAMR) strategy, which requires only two initial Ritz vectors without compromising the accuracy of the simulation. This solving method is tested in the case of shear of a stiffened panel.
\end{abstract}

Keywords: Post-buckling, stiffened structures, model order reduction, Ritz basis completion

\section{Introduction}

The stiffened structures used in a variety of applications (especially aeronautics) are likely to buckle locally without compromising their integrity. By allowing this local buckling of structures while preventing permanent deformations, their weight can be further reduced. For this reason, interest in post-buckling has grown recently, leading to the study of induced phenomena, e.g. composite skin/stiffener debonding [1, 2, 3. In these studies or during preliminary and advanced design phases, the post-buckling simulation of structures is needed not only once but several times within optimization procedures [4, 5].

Analytical and semi-analytical approaches have demonstrated their relevance in the preliminary design phases through their low computational costs and the establishment of closed-form solutions, which are suited to parametric analysis and optimization [6, 7]. However, complex geometries and loadings, material properties and multi-scale phenomena restrict their use in advanced design phases. Although an advanced description of a stiffened panel has been developed by considering elastic restraints due to the stiffeners [7],

\footnotetext{
* Corresponding author

** Principal corresponding author
} 
the buckling interactions between neighbouring panels are not accounted for in these approaches for the moment.

In contrast, the versatility of the finite element method enables it to tackle a wide range of issues. However, the computational cost is much higher and may prevent the simulation of the post-buckling of large scale structures [2, 8]. This is the reason why many works have sought to develop efficient solving strategies. Among these are the multiscale approaches $[8,9,10$ and the model reduction techniques $[11,12,13,14,15$. In this paper, a projection-based model reduction strategy (i.e. based on the projection of the unknown vector on a Ritz basis) for post-buckling analysis is proposed. Attention is paid to the accuracy of the resulting approximation and to the cost of setting up the post-buckling Ritz basis.

According to aeronautical requirements regarding post-buckling of structures, it is assumed that the targeted applications remain in the framework of the early stage of post-buckling and verify the hypothesis of small strains and moderated rotations. So the authors focus on a way to use mechanical knowledge of the early stage post-buckling in order to build an efficient Ritz basis, i.e. a small Ritz basis able to represent accurately the solution of the considered problem. In a first section, the state of the art of this knowledge is reviewed in the light of the (semi-)analytical methods. After having recalled the principles of projection-based model reduction and methods to control the approximation error, an adaptive model reduction strategy is proposed. The second section presents the validation of the assumptions and the evaluation of the computational performance of the strategy. The behaviour of the strategy is described in the case of a simple plate. An application to a stiffened panel is then proposed, which is more representative of industrial needs.

\section{Literature review}

\subsection{Understanding of the post-buckling behaviour of structures: closed-form solutions}

In a first stage of post-buckling, the non-linearity is purely due to geometric effects. This type of nonlinearity is accounted for in mechanical modelling by the assumption of large displacements, small strains and moderate rotations. The non-linear system of equations that governs the static equilibrium of domain $\Omega$ is commonly built from the variational formulation of the potential energy $\Pi_{t o t}(1)$ (where $\Pi_{e x t}$ is the energy of the external forces).

$$
\delta \Pi_{t o t}(u)=\int_{\Omega} \underline{\underline{\sigma}}: \underline{\underline{\delta \varepsilon}}(u) d \omega-\delta \Pi_{e x t}
$$

Green-Lagrange strains $\underline{\underline{\varepsilon}}(2)$ and conjugated stresses $\underline{\underline{\sigma}}$ by the Hooke tensor $\underline{\underline{\underline{\underline{C}}}}(3)$ are used in the framework of large displacements, small strains and moderate rotations.

$$
\underline{\underline{\varepsilon}}=\frac{1}{2}\left(\nabla u+\nabla^{T} u\right)+\nabla u \nabla^{T} u
$$




$$
\underline{\underline{\sigma}}=\underline{\underline{\underline{C}}}: \underline{\underline{\varepsilon}}
$$

In the case of plates, the in-plane stresses may also be approximated by the Airy stress functions $\chi(x, y)$ (4), as observed in the literature [7, 16].

$$
\underline{\underline{\sigma}}=\left(\begin{array}{ccc}
\partial_{11} \chi(x, y) & -\partial_{12} \chi(x, y) & 0 \\
-\partial_{12} \chi(x, y) & \partial_{22} \chi(x, y) & 0 \\
0 & 0 & 0
\end{array}\right)
$$

$\partial_{\alpha \beta} g(x, y)$ being the derivative of $\mathrm{g}$ according to $\alpha$ and $\beta$, which represent either $x$ or $y$. Approximate expressions of displacement field and/or stress state, that are solutions of equation (1), were sought for simplified configurations by means of analytical Ritz formulations. Besides providing an understanding of the post-buckling, these analytical approximations are based on assumptions that could guide the development of finite element model reduction. Therefore, an insight is given into how a closed-form solution is established for the post-buckling of panels [17, 6, 18, 7].

Generally, the governing equations are derived analytically while the solution is obtained numerically for reasons of non-linearity. The following assumption is taken to hold in the early stage of post-buckling: The post-buckling equilibrium stress/displacement state is close to the fundamental equilibrium state and is obtained by a small variation of the stress/displacement field [17].

Solving the governing equation by the Ritz method has led to various approaches. In the work by Koiter et al. [17] on the post-buckling of a cylindrical panel under axial compression, the variation of the full displacement field due to post-buckling is approximated and the problem is solved with displacement unknowns only. In the case of formulations of flat panel problems [18, the Airy stress function $\chi(x, y)$ and the out-of-plane displacement $w(x, y)$ are the unknowns that respectively enable the in-plane and the out-of-plane variations of stress/displacement fields due to post-buckling to be described. The selected unknowns are approximated by a finite sum of kinematically (respectively statically) admissible displacement (respectively stress) functions.

The accuracy of Ritz methods depends on the number of terms in the sum, as observed by Bisagni et al. 18]. However, increasing the number of terms may reduce the efficiency of the method. In contrast, it is interesting to note that Koiter [19, 17, and Vescovini et al. 7] stated the following decomposition of the variation of displacement field due to post-buckling $\delta u_{P B}$.

$$
\delta u_{P B}=a \times u_{B}+\delta \bar{u}
$$

where $a$ is the amplitude of the buckling mode $u_{B}$ and $\delta \bar{u}$ is a small higher order variation. In accordance with this assumption, it was shown by Vescovini et al. [7] that neglecting $\delta \bar{u}$ still led to a small error (less than 
$7 \%$ ) in the load-displacement curves. In consequence, the dependence of the accuracy of the approximation on the number of Ritz functions is reduced, as is the total number of unknowns (multiplier factors of the Ritz functions).

Despite the good agreement shown between the maximum values of displacement and stress fields computed by the finite element and semi-analytical methods when neglecting the higher order variation terms [7, it should be noted that their distributions are significantly different. This may have an impact on the design of structures. The higher order variations of displacement field are thus of major importance when it comes to accounting for the stress redistribution in post-buckling. The semi-analytical methods are applied in the range of the early post-buckling stage, which still allows more than twice the critical buckling load to be reached in practice 17, 18, 17]. Finally, the review of semi-analytical methods highlights the assumptions on stress and displacement fields that have enabled the post-buckling behaviour of plates and shells to be predicted efficiently. The Ritz method is indeed successfully rationalized (Two terms are easely computed and can reproduce the solution with relatively good accuracy), which simplifies the user's choice of truncation order of the Ritz functions and reduces the computation cost. From the standpoint of the finite element method, these considerations could be useful to reduce the size of the models. In the next section, a method is presented which enables the use of a priori knowledge from the semi-analytical methods to reduce the finite element model.

\subsection{Model reduction techniques in non-linear structural finite element analysis}

In structural mechanics, the finite element discretization of the governing equations arises from the Galerkin method applied to the variational formulation of the potential energy (1) 20]. The approximation of the displacement field by means of piecewise polynomial interpolation functions results in the matrix form of the static equilibrium at time increment $t_{n}(6)$.

$$
\underline{\underline{K}}\left(\underline{U}_{\mid t_{n}}\right) \cdot \underline{U}_{\mid t_{n}}=\underline{F}_{e x t}
$$

Due to geometric non-linearities, the stiffness matrix $\underline{\underline{K}}$ depends on the displacement $\underline{U}$. Hence a minimization problem can be written, the objective function of which is a norm of residual forces (Newton-Raphson method), which leads to the iterative solving of the tangent system (7) until a convergence condition is fulfilled. Index $i$ denotes iteration $i$.

$$
\underline{\underline{K_{t}}}(\underline{U}) \cdot \underline{\delta U^{i}}=-\underline{R}(\underline{U})
$$

The projection-based model reduction techniques have been applied to various non-linear problems [21, 22, 15, 14, 11. By using the a priori knowledge of the problems, they aim to reduce the size of the matrix systems presented so as to speed up problem solving. The displacement vector and its increments are basically approximated by a combination of well-chosen Ritz vectors (extracted from the a priori knowledge). The 
vectors form a reduced basis (also called the Ritz basis) $\underline{\underline{C}}$ that spans a subspace $\operatorname{Im}(\underline{\underline{C}})$ of $\mathbb{R}^{N}, N$ being the number of degrees of freedom of the initial FE problem. The approximate displacement and displacement increment are denoted $\underline{U}_{C}$ and $\underline{\delta U_{C}}$ and are a linear combination of the basis vectors:

$$
\left\{\begin{array}{l}
\underline{U}_{C}=\underline{\underline{C}} \cdot \underline{\alpha} \\
\underline{\delta U}_{C}=\underline{\underline{C}} \cdot \underline{\delta \alpha} \\
\alpha \text { is the vector of generalized coordinates }
\end{array}\right.
$$

According to the Galerkin procedure, the residual forces must be orthogonal to the space spanned by the reduced basis. After approximating $\underline{\delta U}$ by $\underline{\delta U}$ and orthogonalizing the residual forces with respect to $\underline{\underline{C}}$, the reduced tangent system reads:

$$
\underline{\underline{C}}^{T} \underline{\underline{K_{t}}}(\underline{U}) \underline{\underline{C}} \cdot \underline{\delta \alpha}^{i}=-\underline{\underline{C}}^{T} \underline{R}(U)
$$

The dimension of the reduced tangent system is equal to the number of Ritz-basis vectors, which is usually several orders of magnitude smaller than $N$ and makes the system solving less time consuming. However, since $\underline{\delta U_{C}^{i}}$ is not equal to $\underline{\delta U^{i}}$, the tangent system of iteration $\mathrm{i}+1$ is not based on the same operators $\underline{\underline{K_{t}}}\left(\underline{U}^{i}\right)$ and $\underline{R}\left(\underline{U}^{i}\right)$, whether iteration i was performed in the reduced subspace $\operatorname{Im}(\underline{\underline{C}})$ or in the full $\mathrm{FE}$ space $\mathbb{R}^{N}$. For this reason the convergence rate of the reduced Newton procedure may be different from that of a full or a modified Newton procedure. Hence the projection-based model reduction techniques not only affect the size of the tangent system, but also the number of iterations of the minimization procedure of the residual forces.

\section{Building a reduced basis}

Building a reduced basis is of fundamental importance in finite element model reduction, as the choice of Ritz functions is in analytical methods. Reduced bases from modal analysis have demonstrated their ability to reduce models in structural dynamic analysis [12, 22. Several reduced bases have been proposed for geometrically non-linear model analysis [23, 24, 15]. In these fields, basis vectors, also called Ritz vectors, are often chosen because of physical considerations but it is also possible to use experience gained over similar model simulations or live test measurements. In order to extract an optimal reduced basis from such a large set of vectors, usually called a snapshot, the proper orthogonal decomposition (POD) computes a truncated singular value decomposition (SVD) [25, 11. Whatever the method, the reduced basis influences the accuracy of the approximation. In the incremental-iterative solving method for non-linear mechanical problems, the multiplicity of approximated equilibrium states makes it more difficult to generate, a priori, reduced bases with small cardinality. 


\section{Definition of the approximation error}

The accuracy of the reduced model, as well as the accuracy of any approximate solving procedure, is defined by the difference between the solution (generally unknown) and its approximation:

$$
\text { solution error }=\left\|\underline{U}-\underline{U_{C}}\right\|
$$

The solution vector belongs to the initial FE space of dimension N. This space may be decomposed into two supplementary subspaces:

$$
\left\{\begin{array}{l}
\mathbb{R}^{N}=\operatorname{Im}(\underline{\underline{C}}) \oplus \operatorname{Im}(\underline{\underline{C}})^{\perp} \\
\text { where }{ }^{\perp} \text { defines an orthogonality condition in } \mathbb{R}^{N}
\end{array}\right.
$$

In other words, the solution vector is decomposed as follows:

$$
\left\{\begin{array}{l}
\underline{U}=\underline{U_{C}}+\underline{U_{K}} \\
\underline{U_{C}} \in \operatorname{Im}(C) \\
\underline{U_{K}} \in \operatorname{Im}(C)^{\perp}
\end{array}\right.
$$

The solution error is directly linked to the unknown part $\underline{U_{K}}$. Practically, in order to quantify the solution error, the relative norm of the residual forces can be computed:

$$
e=\frac{\|\underline{R}\|}{\| \underline{\underline{F}_{e x t} \|}}
$$

Since it tends towards zero as the solution approaches equilibrium, the relative norm of the residual forces is often used in a convergence criterion of iterative solving procedure (Newton like) [20]. A threshold generally enables to set the desired level of accuracy to be reached, which is refered to in Newton's solvers as the convergence parameter $\epsilon_{\text {New }}$. However, due to model reduction, the relative norm of residual forces resulting from the approximate solution $\underline{U_{C}}$ may not converge towards zero. This could prevent from reaching the desired level of accuracy and is discussed in what follows.

\section{Controlling the approximation error}

In order to control the approximation error, i.e. to fall below the convergence parameter of the relative norm of the residual forces, it is mandatory to enrich the reduced basis. For that purpose, it is necessary to compute a displacement vector belonging to $\operatorname{Im}(\underline{\underline{C}})^{\perp}\left(\underline{U_{K}}\right.$ or an approximation $\left.\widetilde{U_{K}}\right)$, at minimal cost, as seldom as possible. Once computed and normalized, it can be added to the reduced basis $\underline{\underline{C}}$.

Two types of completion procedures can be identified in the literature.

- a posteriori completion is based on a full Newton-Raphson increment. The solution $\underline{\Delta U}$ of this increment is split afterwards into $\underline{\Delta U_{C}}$ and $\underline{\Delta U_{K}}$ by projection and the latter is normalized and added to the reduced basis [21], or the basis vectors are simply updated before the next reduced increment 24, 15]. 
- on the fly completion consists of enriching the reduced basis during a Newton iteration. Basically, $\underline{\delta U_{K}}$ is computed from the projection of the solution $\underline{\delta U}$ of the full tangent system of the iteration and normalized to complete the reduced basis. But in the case where $\underline{\underline{K}}_{T}$ is symmetric positive definite, a more efficient procedure is proposed by Kerfriden et al. [11]. The solution space is split into two supplementary subspaces $\operatorname{Im}(\underline{\underline{C}})$ and $\operatorname{Im}(\underline{\underline{C}})^{\perp_{K}}$. In this way, the reduced tangent system $(9)$ and the projected tangent system (14b) (where $P$ is a classical projector 14a) are uncoupled and are solved together instead of solving the full tangent system.

$$
\begin{aligned}
& \underline{\underline{P}}=\underline{\underline{I}}-\underline{\underline{C}}\left(\underline{\underline{C}}^{T} \underline{\underline{K_{t}}} \underline{\underline{\underline{C}}}\right)^{-1} \underline{\underline{C}}^{T} \\
& \underline{\underline{P}}^{T} \underline{\underline{K}} T \underline{\underline{P}} \cdot \underline{\delta U_{K}}=-\underline{\underline{P}}^{T} \underline{R}
\end{aligned}
$$

$\delta U_{K}$ is directly and efficiently computed by solving $14 \mathrm{~b}$ by a conjugate gradient, because the projection framework ensures good conditioning properties.

Each completion procedure has an adapted triggering criterion. The non-compliance of the normalized residual forces (13) with desired level of accuracy $\left(e>\epsilon_{\text {New }}\right)$ after a reduced Newton increment, which converged towards approximate solution $\underline{U_{C}}$, is basically the triggering criterion suited to a posteriori completions. Some variations make use of the current stiffness parameter [24, 15] to avoid completions in slightly non-linear areas. The second strategy relies on the comparison between the normalized full residual forces 13 and the normalized reduced residual forces 15, which is the relative norm of the residual forces projected into the reduced subspace $\operatorname{Im}(\underline{\underline{C}})$. It was initially designed for on the fly completion by [1].

$$
e_{C}=\frac{\left\|\underline{\underline{C}}^{T} \underline{R}\right\|}{\left\|\underline{\underline{C}}^{T} \underline{F}_{E x t}\right\|}
$$

A parameter $k$ is defined such that the completion is triggered when $e_{C}<k \times e$. This criterion is particularly suited to on the fly completion since it reflects completeness of the reduced basis during Newton iterations. As illustrated in Fig 1, the larger $k$ is, the earlier a completion enhances an iteration. But a compromise must be found to minimize the total number of completions.

Both completion approaches aim to control the approximation error resulting from projection-based model reduction but the main difference is a numerical issue. In this field, on the fly completion has the advantage of enhancing one iteration at a time whereas a posteriori completion runs an entire full NewtonRaphson increment. In addition, the use of a conjugate gradient to solve the enhanced linear system of the on the fly completion only requires matrix products. Furthermore, Kerfriden et al. [11] demonstrated that just a coarse approximation of $\underline{\delta U_{K}}$ was enough to complete the reduced basis, thus minimizing the number of gradient iterations.

Nevertheless, the computation performance depends on the total number of completions required during the whole calculation. It would be interesting to compare on the fly and a posteriori completions for different applications. 


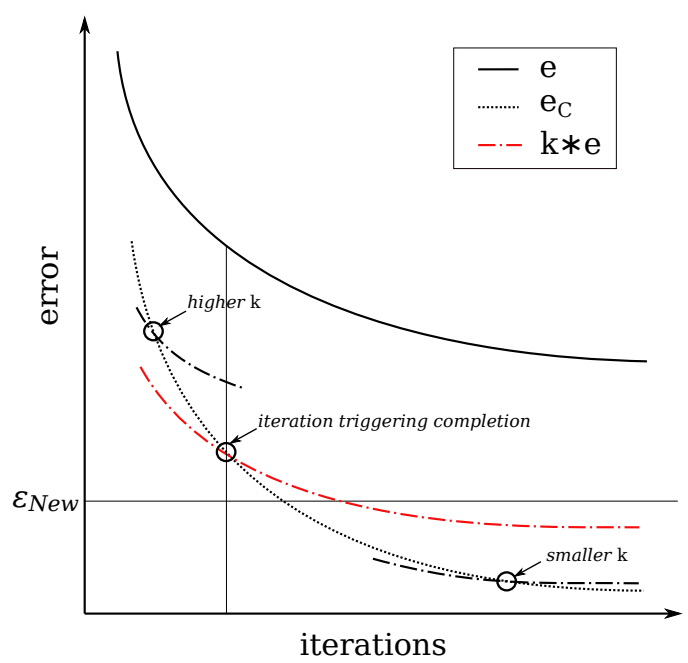

Figure 1: Schematic representation of the completion criterion and the influence of $k$.

Completion procedures can be associated with a control of the number of basis vectors. Controlling the size of the reduced basis prevents an accumulation of basis vectors over iterations, which leads to a loss of computational efficiency. A wide range of procedures could be proposed. Kerfriden et al. [11 have proposed a reduced basis management involving two levels:

- At the level of a time increment, completion vectors $\underline{\delta U_{K}}$ are accumulated in a sub-basis $\underline{\underline{C}}_{C G}($ $\left.\underline{\underline{C}}_{\text {increment }}=\left[\begin{array}{ll}\underline{\underline{C}} & \underline{\underline{C}}_{C G}\end{array}\right]\right)$ whose basis vectors are $\underline{\underline{K}}_{T}$-orthogonal to each other. Once the increment has converged, the incremental displacement vector is orthonormalized with respect to the reduced basis and is added to the latter as a single completion vector. It is worth noting that such treatment applies only to a modified Newton iterative procedure for $\underline{\underline{K}}_{T}$-orthogonality considerations.

- At the level of several time increments, the size of the reduced basis is kept below a user-defined maximum. When this maximum is reached, the over-sized reduced basis is considered as a snapshot of a POD procedure.

In spite of the expected benefits of controlling the size of the reduced basis, it has been demonstrated in 11. that such procedures may lead to extra completions when the maximum size is set too small. Extracting a part of a reduced basis may indeed result in a loss of information that is important for the representation of the next equilibrium state.

\section{Applications to post-buckling analysis}

In the literature, projection-based model reduction has been applied to post-buckling analysis. Kling et al. 15] proposed a hybrid subspace whose components are the last converged full increment solution, some buckling modes and a few path derivatives calculated using static perturbation techniques. Earlier, Noor 
and Peters [13] had proposed using path derivatives alone as a reduced basis in the case of geometrically nonlinear model reductions. Both reduced bases were coupled with an a posteriori completion procedure that led to the update of their components to control the error. Nagy and Konig 23 focused on a superposition of buckling modes to reduce the size of mildly non-linear post-buckling problems. Despite not using any completion procedure, they found good agreement in terms of load-displacement curves.

The accuracy of these strategies, as well as the number of a posteriori completions and updates of reduced basis required, depend on the truncation of the buckling mode basis and/or the order of the path derivatives. The same holds for computational performance and a compromise must be found between increasing the size of the initial reduced basis and completing or updating it.

\section{Low cost initial reduced basis and on-the-fly adaptive procedure for post-buckling model reduction}

Given the considerations mentioned previously, the purpose of this paper is to take a step back to a simpler description of post-buckling in projection-based model reduction approaches, inspired by the asumptions on post-buckling displacement field made in semi-analytical methods (i.e. the post-buckling state can be approximate by a combination of the fundamental state, the result of linear buckling analysis and higher order variation terms representing stress redistribution). Also, the objective of the current section is to propose a rationalized and efficient model reduction strategy for solving post-buckling problems, referred to as the Post-Buckling Adaptive Model Reduction (PBAMR).

A low-cost initial reduced basis is built from the understanding of post-buckling behaviour in 1.1 . The displacement field in the early stage of post-buckling has indeed been described as a combination of the displacement field of the fundamental equilibrium state, the linear buckling mode and a higher order variation term. In consequence, in addition to the normalized fundamental solution $\underline{U}_{\mid t_{0}} /\left|\underline{U}_{\mid t_{0}}\right| \mid$, which is easily computed, the proposed strategy consists of keeping only the first buckling mode, unlike Nagy et al. [23, who considered a truncation of the buckling mode basis. The consequences of this choice on the field of applicability of the strategy is discussed later in this section. Therefore the initial reduced basis (16) is made of two vectors and its computational cost is reduced in comparison to existing strategies.

$$
\underline{\underline{C}}_{\text {init }}=\left[\underline{U}_{\mid t_{0}} /\left|\underline{U}_{\mid t_{0}}\right| \mid \underline{U}_{B}\right]
$$

The initial reduced basis is associated with an on the fly completion procedure to account for stress redistributions. This completion procedure is chosen for the reasons set out below:

- It is more flexible (one enhanced iteration at a time).

- As the stress redistribution occurs between consecutive equilibria of the post-buckling stage, completions may be required over several increments. 
The higher order variation of displacement is thus evaluated during the simulation. To the authors' knowledge, although it has recently demonstrated its relavance in the case of damageable lattice structures [1], on the fly completion has not been applied either to post-buckling model reduction, or to geometrically non-linear analysis yet.

The PBAMR strategy is based on a Newton incremental-iterative procedure. In the general algorithm presented in Alg 1. the projection of the tangent system and the on the fly completion were basically added to a classical full Newton-Raphson algorithm. For this reason, the computational performances of the PBAMR are compared to those of a full Newton-Raphson procedure in section 3 . It should be noted that while the completion triggering criterion involves both the normalized reduced residual forces and the normalized full residual forces (line 5 of Alg,1), the convergence criterion remains based on the comparison of the normalized full residual forces and the convergence parameter (line 4 of $\mathrm{Alg}$, 1), as is the case of the convergence criterion of classical Newton scheme. Having such convergence criterion makes the PBAMR strategy as accurate in terms of approximation error as a classical Newton procedure for each time increment, whatever the initial reduced basis. The issue rather concerns the number of iterations and completions required to reach the desired accuracy, which may depend on the initial reduced basis and the completion parameter $k$.

In Alg, 1, the fundamental solution, line 1, may either be computed from a previous increment of the non-linear procedure or from a linear analysis. In the initialization of the incremental procedure line 3 , the displacement can likewise be set to:

- null displacements and forces, e.g. if non-linear behaviour is expected before buckling.

- or to the first step solution $\left(\underline{U}_{\mid t_{0}}\right)$

- or to $\lambda_{c r} \times \underline{U}_{\mid t_{0}}$ in order to start analysis close to the bifurcation point.

In this paper, as few completions were expected, a first approach was to let the reduced basis grow over completions. Consequently, instead of controlling the size of the reduced basis line 7, an orthonormalization of the completion vectors with respect to $\underline{\underline{C}}$ was implemented, line 6 . Moreover, the components of the displacement field in the reduced basis (generalized coordinates) can be monitored in order to better understand the behaviour of the strategy and the role of additional basis vectors.

Finally, the PBAMR strategy is presently designed to represent post-buckling states by using the displacement field of the fundamental equilibrium state and the first buckling mode, which are gathered in the initial reduced basis, and some additional vectors, which are computed by the on the fly completion. In the case of post-buckling mode-switching from a buckling mode to another at a secondary bifurcation point [26], the proposed initial reduced basis becomes theoreticaly obsolete. This point is not handled by semianalytical methods based on the asumption (5), but the present adaptive strategy still could complete the reduced basis to represent the post-buckling displacement field after a secondary bifurcation point. However, 


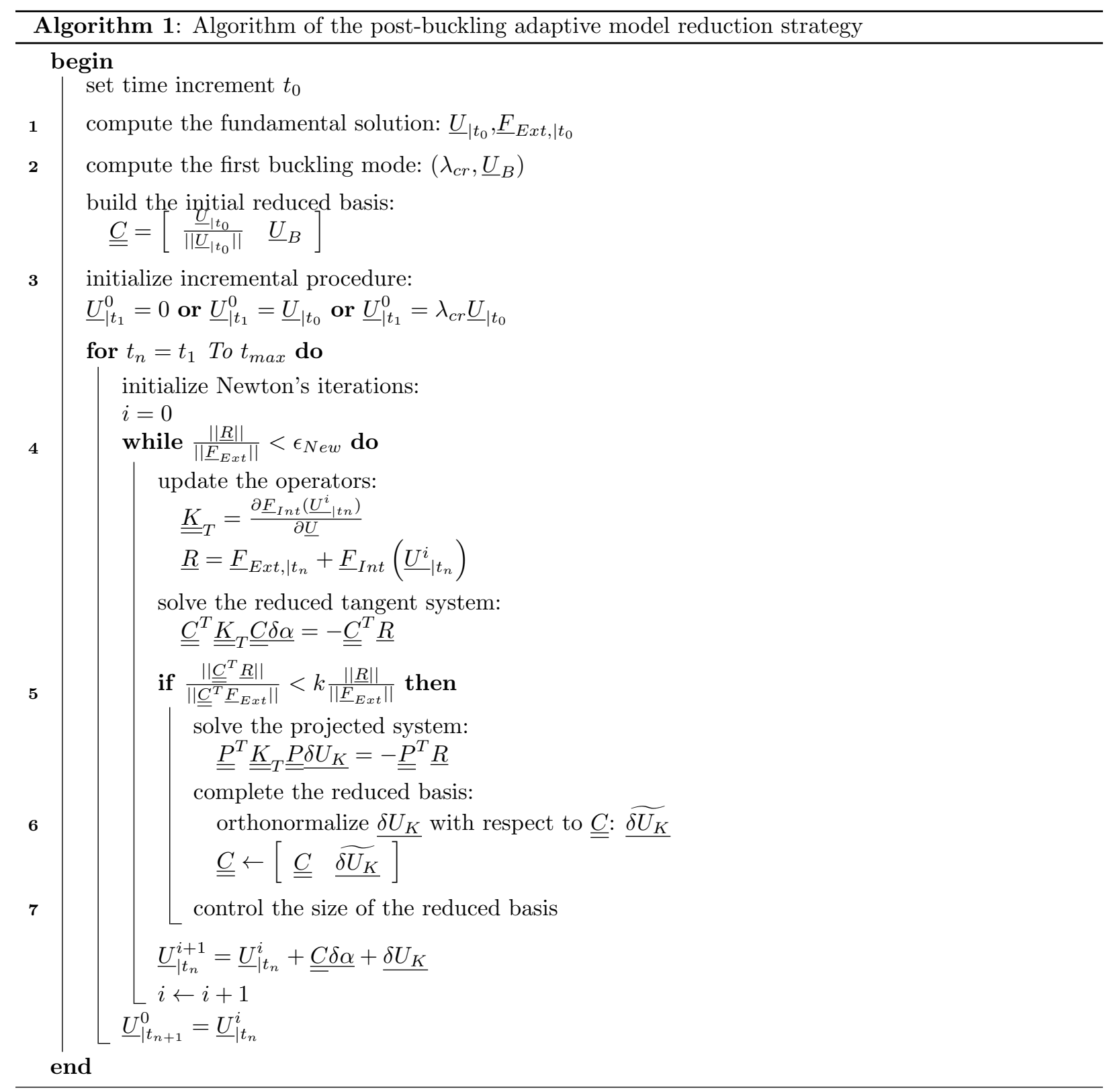


in this case the number of completions is expected to be high. It is indeed generally not accurate to represent a post-buckling displacement field by the combination of a buckling mode vector not corresponding to the observed buckling mode and additional vectors that account for stress redistribution in the first buckling mode.

This raises the issues of bifurcation point detection [27] and update of the reduced basis [15, that are not addressed in this paper and would require further developments in order to keep benefit from a rationalized description of the post-buckling displacement field after a secondary bifurcation point. Another strategy could consist in building the initial reduced basis with all the buckling modes whose critical load factor could be reached in the range of loading. In this case, the non-linearity of the load/displacement curve could introduce error in the expected applied load [20]. Thus, the present PBAMR strategy will not be applied in the case of mode switching and the scope of this paper is limited to the demonstration of the relevance of the PBAMR strategy, while remaining on the same bifurcation branch.

\section{Implementation and evaluation of the proposed strategy}

In the present section, the evaluation of the PBAMR strategy relies on two finite element models (FEM). A first simple model of a rectangular plate is used to gain a better understanding of the behaviour of the strategy. The second FEM, which tries to represent the targeted industrial applications, is aimed at providing insight into the computational performance.

The strategy was implemented in a finite element code using the programming language Python [28] (http://www.scipy.org) and which was developed for the purposes of the present research. The Mindlin thick plate kinematics was chosen together with a quadratic interpolation and reduced numerical integration. A total lagrangian formulation was adopted with Green-Lagrange strain and Piola-Kirchhoff II stress measurements. Such FE approximation is commonly used in the field of post-buckling analysis of stiffened structures [2].

Because of the limits of the research code, modelling was restricted to flat plates. In order to trigger the buckling of the numerical models, the displacement field was perturbed by a buckling mode displacement having an amplitude of half the plate thickness.

\subsection{Behaviour of the proposed strategy and parametric study}

The following numerical study focuses on a rectangular plate model whose properties are detailed in Tab 1. The behaviour analysis and the parametric study of the strategy are based on the case of pure shear loading Fig 2 applied by the mean of boundary conditions on the four edges of the plate as follow:

- $u(x, y)=0.001 \times y$

- $v(x, y)=0.001 \times x$ 
- $w(x, y)=\theta_{1}(x, y)=\theta_{2}(x, y)=0$

\begin{tabular}{ccc|ccc}
\hline Geometry & & & \multicolumn{3}{|c}{ Mechanical properties } \\
\hline length & 1000 & $\mathrm{~mm}$ & $\mathrm{E}$ & 70000 & $\mathrm{MPa}$ \\
width & 700 & $\mathrm{~mm}$ & $\nu$ & 0.33 & \\
thickness & 7 & $\mathrm{~mm}$ & & & \\
\hline
\end{tabular}

Table 1: Geometrical and mechanical properties of the test plate

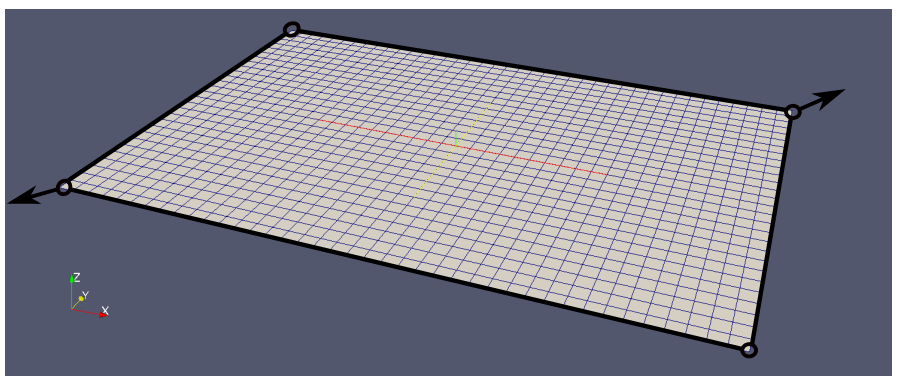

Figure 2: Load case definition: the edges are rigid and articulated at the corners

Validation of the results using a commercial code

A comparison with a commercial FE software (Abaqus) is given in order to assess the quality of the research FE code and the implemented solving procedures. The model has been discretized in 20 elements in lentgh and 14 elements in width. The buckling load factors are first computed on a pre-stressed state obtained by applying $10 \%$ of the maximum boundary conditions previously described. The small relative error reported in Tab 2 and the strong similarities of the shapes of the corresponding buckling modes Fig 3 validate the buckling analysis performed by the research code.

\begin{tabular}{cccc}
\hline & Abaqus & Research code & Relative error \\
\hline$\lambda_{1}$ & 4.55 & 4.75 & $4.4 \%$ \\
$\lambda_{2}$ & 4.78 & 4.99 & $4.4 \%$ \\
$\lambda_{3}$ & 7.95 & 8.29 & $4.3 \%$ \\
\hline
\end{tabular}

Table 2: Buckling load factors computed on a pre-stressed state obtained by applying $10 \%$ of the total boundary conditions.

The non-linear geometric static analysis is performed in 10 equal increments. As the first buckling load is being exceeded during increment 5 (the imposed displacement reaches $50 \%$ of the maximum displacement, that is more than $\lambda_{1}$ times the imposed displacement of the first increment), the plate is perturbed at the beginning of this increment. Fig 4 and Fig 5 present the distribution of the shear forces and the out-of-plane displacements at increments 6 and 10, which are faithfully represented by the research code, whether it be computed by the full Newton-Raphson procedure or by the PBAMR strategy. 


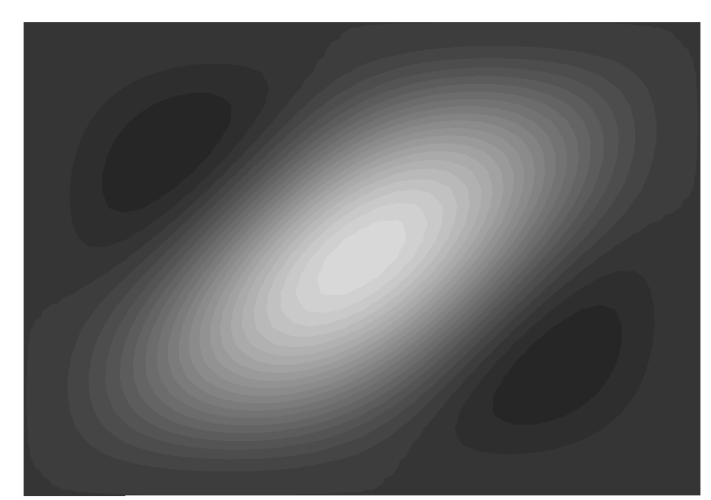

(a) mode 1 by Abaqus

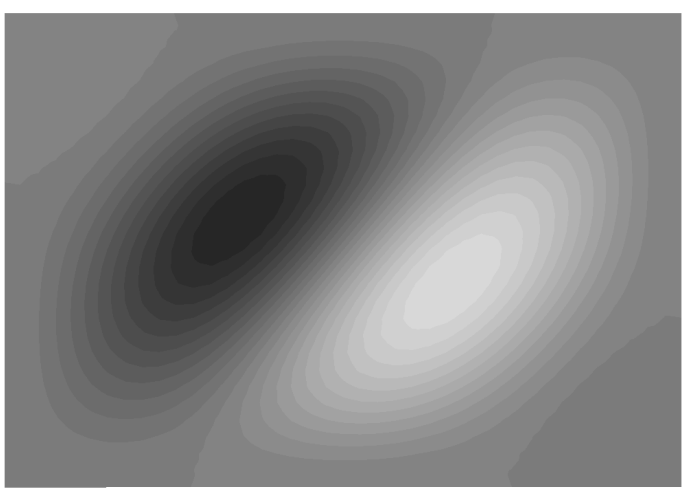

(c) mode 2 by Abaqus

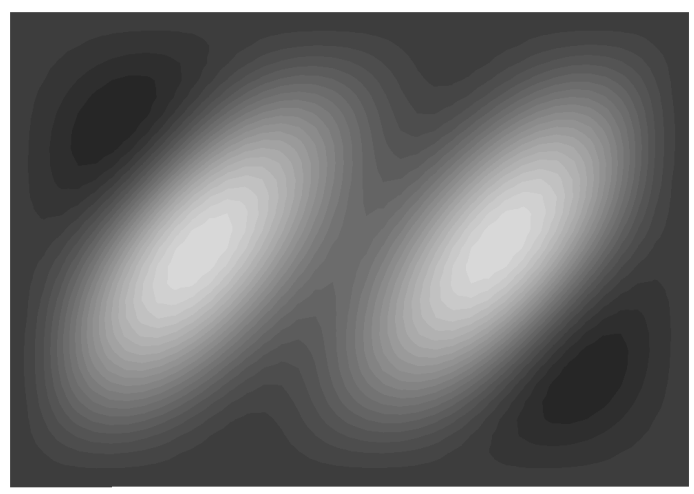

(e) mode 3 by Abaqus

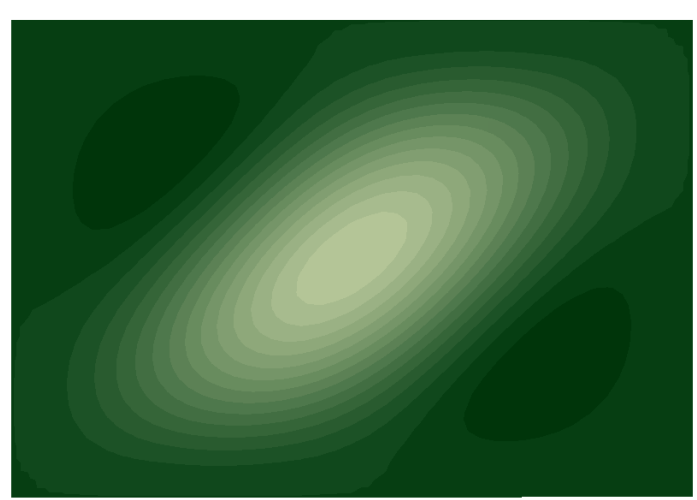

(b) mode 1 by research code

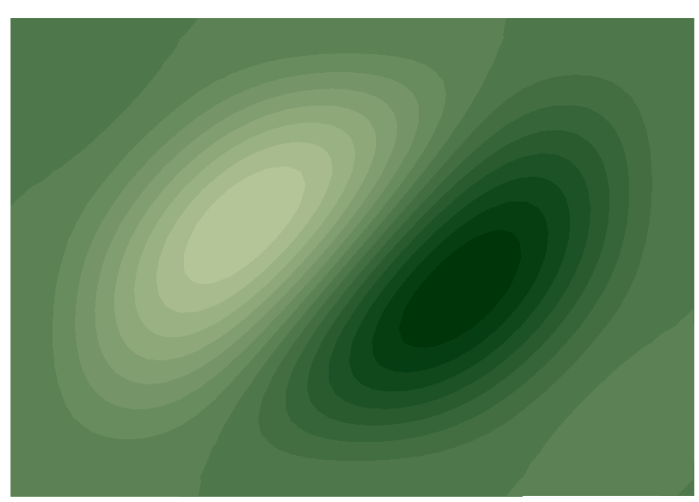

(d) mode 2 by research code

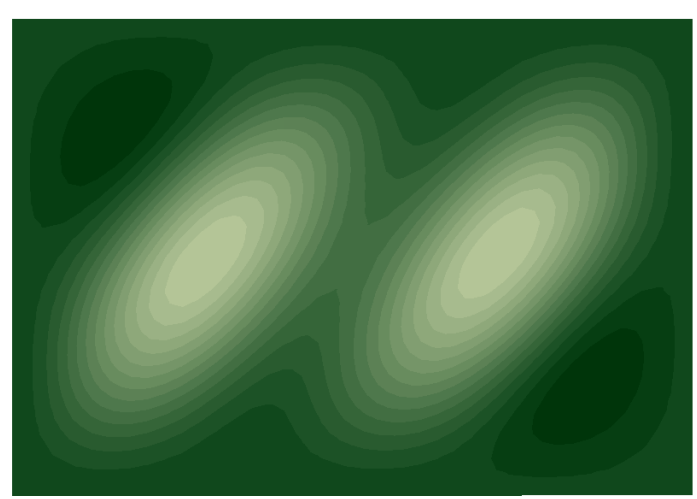

(f) mode 3 by research code

Figure 3: Comparison of the first buckling modes with Abaqus. 


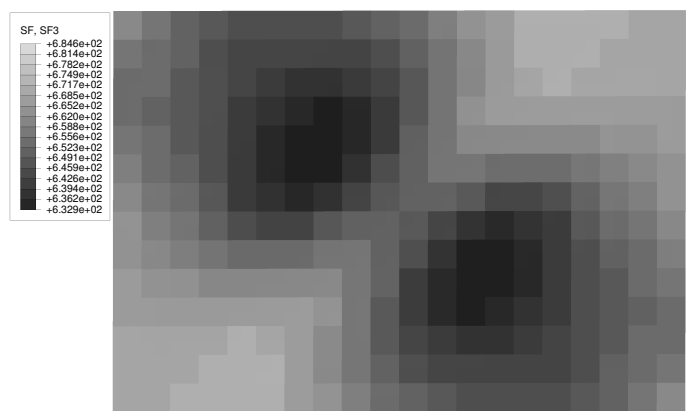

(a) Shear forces at increment 6 by Abaqus

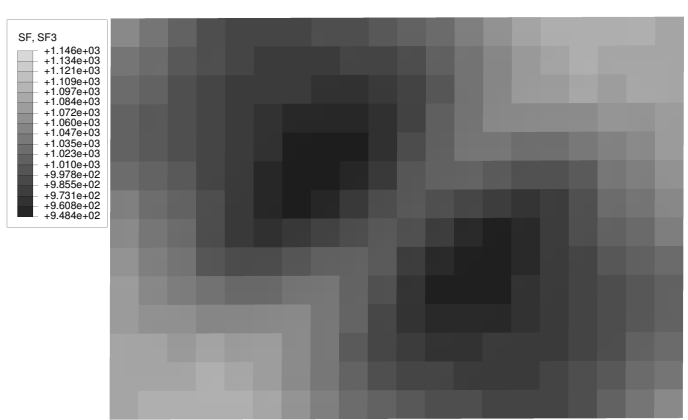

(c) Shear forces at increment 10 by Abaqus

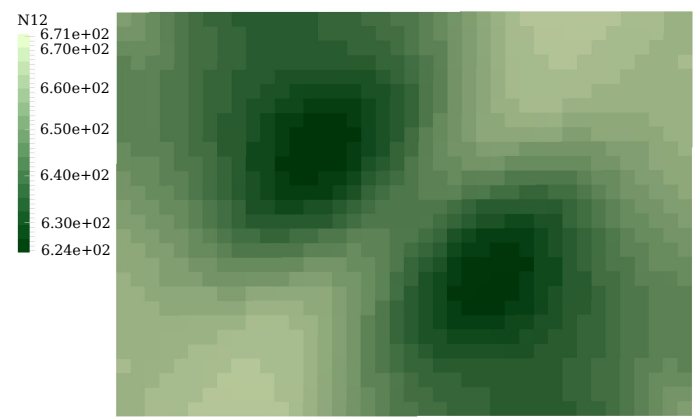

(b) Shear forces at increment 6 by research code

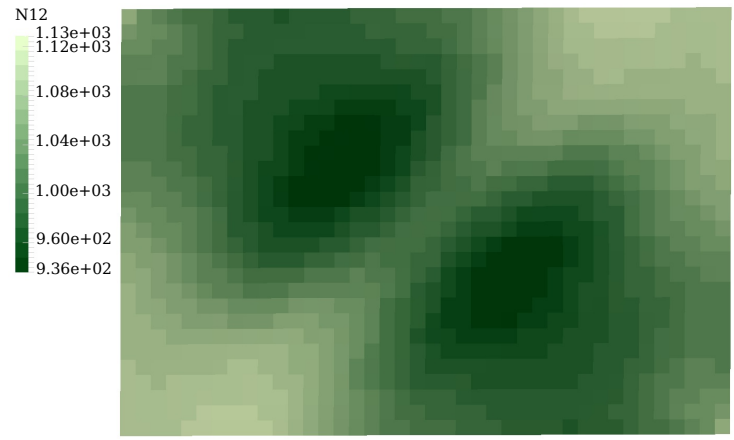

(d) Shear forces at increment 10 by research code

Figure 4: Comparison of the distributions of the shear forces in panel obtained by Abaqus and by the research code (results obtained by Full Newton-Raphson and PBAMR are so close that they are gathered under the name "research code"). It should be noticed that in Abaqus, an element corresponds to one colored cell, whereas four cells per elements are used in the vizualisation of results of research code (one cell per Gauss point). 


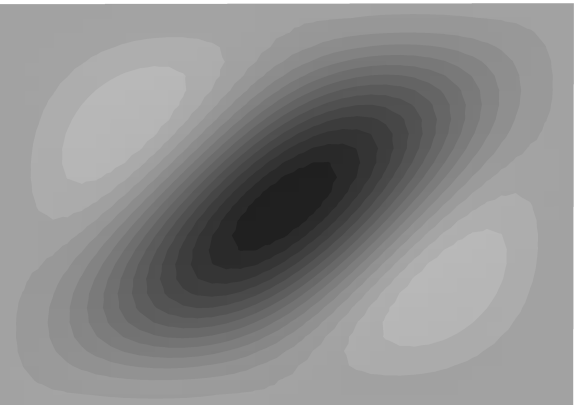

(a) Deflection at increment 6 by Abaqus
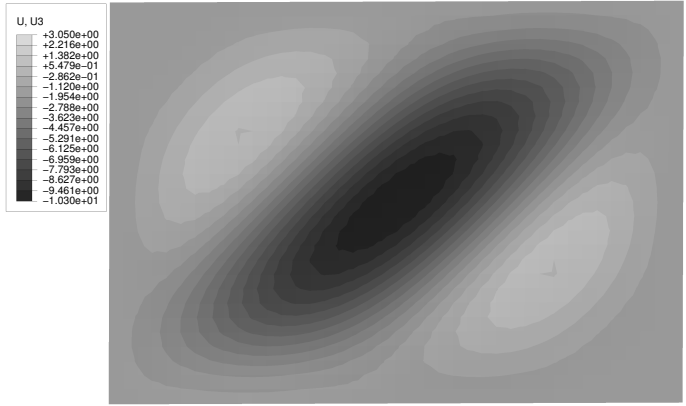

(c) Deflection at increment 10 by Abaqus

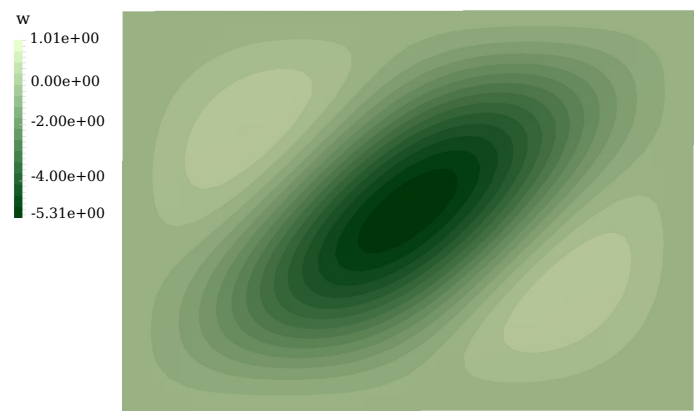

(b) Deflection at increment 6 by research code

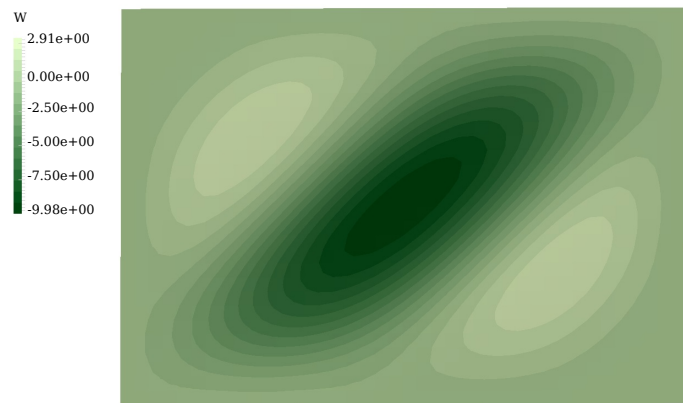

(d) Deflection at increment 10 by research code

Figure 5: Comparison of the deflection of the panel obtained by Abaqus and by the research code.

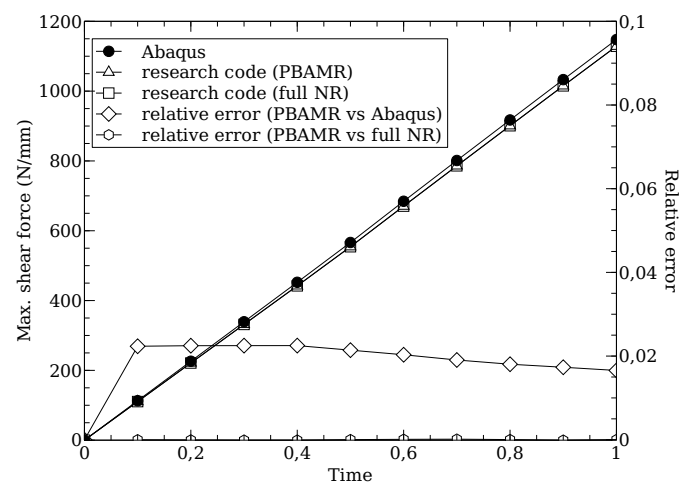

(a)

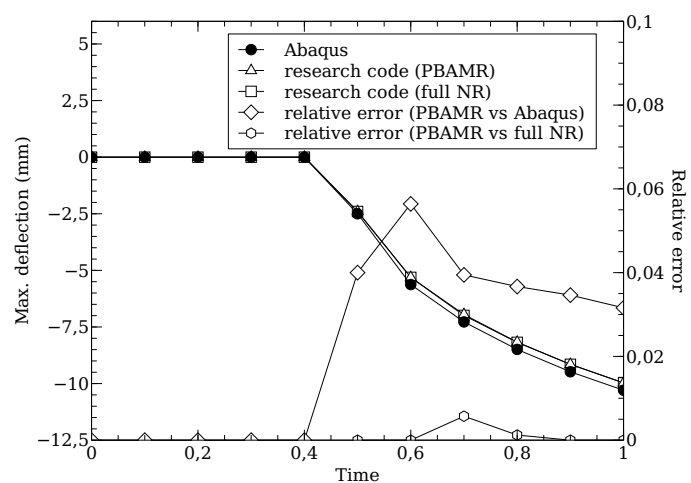

(b)

Figure 6: Comparison of the evolution of the maximum shear force (a) and the evolution of the maximum deflection (b) obtained by Abaqus and by the research code. The relative error is computed between PBAMR and Abaqus results and between PBAMR and full Newton-Raphson results. 
In Fig $6 \mathrm{a}$ and Fig $6 \mathrm{~b}$, the maximum shear forces and deflection were monitored over increments.

On one hand, it results that the research code underestimates the maximum shear forces by less than $2.3 \%$ (1.6\% at increment 10) and the maximum deflection by less than 5.6\% (3.1\% at increment 10). At this level, the relative error is mainly related to the finite element formulation and to numerical considerations. On the other hand, due to the convergence parameter of the PBAMR strategy $\left(\epsilon_{N e w}\right)$, the differences between the results obtained by the PBAMR strategy and the full Newton-Raphson procedure in the research code are negligible, as expected in section 2 .

Finally, the comparison with the results obtained by Abaqus shows not only the accuracy of the research FE code but also the accuracy of the PBAMR strategy as compared with the full Newton-Raphson procedure.

\section{Understanding the behavior of the strategy}

The generalized coordinates of the displacement field were monitored over iterations. Only the generalized coordinates of the out-of-plane displacement field are presented in Fig, 7 . The time increments are represented by vertical dashed lines.

This chart shows the preponderance of the buckling mode in the approximation of the out-of-plane displacement. Five additional basis vectors were computed by on the fly completions in the loading range. This means that only five reduced iterations were enhanced. It can be seen that the generalized coordinates corresponding to additional basis vectors increase with iterations. The sustainability of the first additional basis vectors is thus highlighted. At the same time, the generalized coordinates of the last two basis vectors remain very small. This could indicate that the completion is not achieved in the best possible manner. However, the assumptions about the post-buckling displacement field and the number of completions, as stated in section 2, are validated up to 2.88 times the critical buckling load.

In order to better understand the role of completion, a quantitative study of the shape of the out-of-plane displacement field is proposed.

The shape ratios of the displacement at equilibrium and the buckling mode are compared. The shape ratio is defined as the ratio of a, the minor axis, to $b$, the major axis, of the ellipse drawn by the main buckling blister (see Figs $8 \mathrm{a}$ and 8b), measured at iso-vertical displacement (75\% of the maximum out-ofplane displacement). The shape ratio of the buckling mode is 0.48 . The shape ratio of the displacement at equilibrium decreases with the buckling load factor, as reported in Fig 9 , from 0.43 at $\lambda=1.02 \times \lambda_{c r}$ to 0.33 at $\lambda=2.88 \times \lambda_{c r}$. The additional basis vectors take the shape changes into account as can be observed in Figs $8 \mathrm{c}$ and $8 \mathrm{~d}$. It is interesting to note that the completion modes presented illustrate the lengthening and the narrowing of the buckling waves due to geometrical non-linearity (i.e. stress redistributions).

In order to provide more insight into the role of completions, a model reduction on the initial reduced basis only (i.e. without any completion) is performed. In this procedure, the normalized full residual forces 13 of the convergence criterion are replaced by the normalized reduced residual forces 15 . Since no 


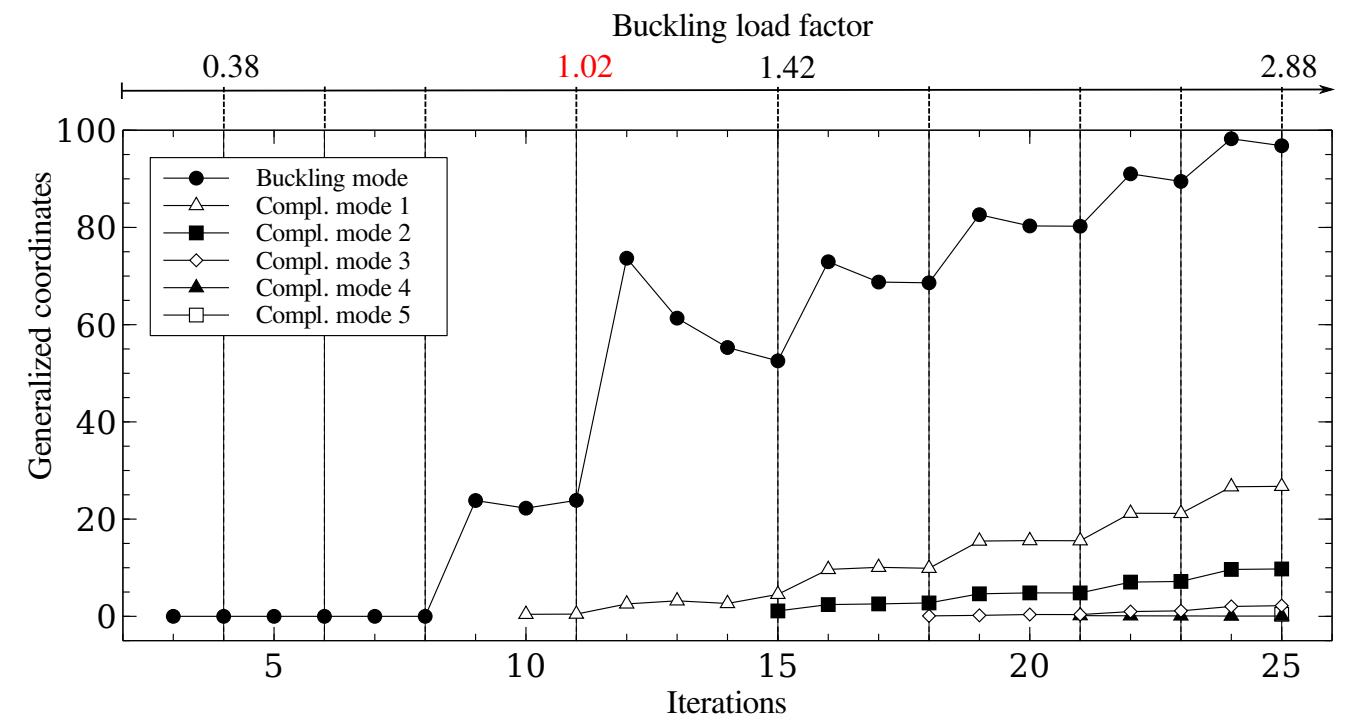

Figure 7: Monitoring of the coordinates of the out-of-plane part of the displacement field in the reduced basis, over iterations and increments of the solving procedure $\left(\epsilon_{N e w}=1 \cdot 10^{-3}\right.$ and $\left.k=10^{-3}\right)$. Vertical dashed lines represent ends of the time increments (equilibrium obtained). The load level is reported in terms of critical buckling load factor at top, for information.

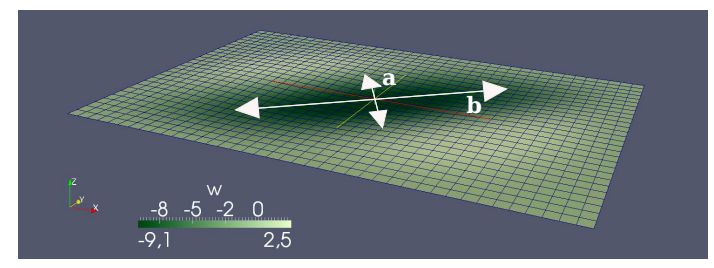

(a)

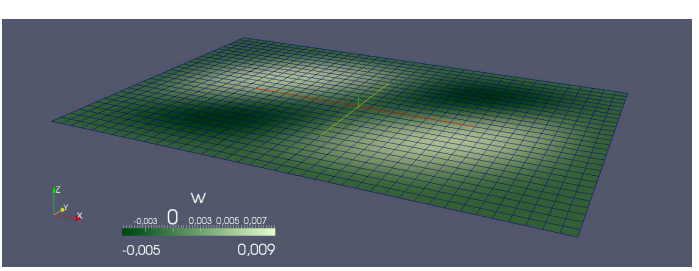

(c)

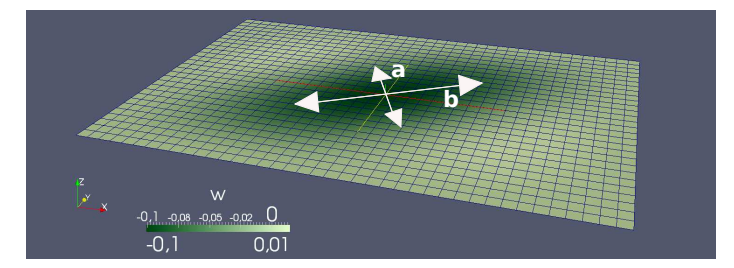

(b)

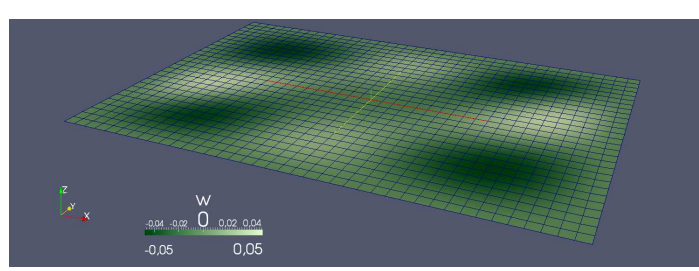

(d)

Figure 8: Solution of the post-buckling analysis of the plate under shear loading (a), first buckling mode (b), and first additional reduced basis vectors $(\mathrm{c}, \mathrm{d})$ calculated by the on the fly completion procedure (Out-of-plane displacement fields).

completions are needed in PBAMR before buckling (because of the linearity of the problem), attention is paid to the post-buckling stage. Fig 10a and Fig 10b report the evolution over increments of maximum shear forces and deflections obtained by the PBAMR strategy and the model reduction without completions. It is noteworthy that not only the relative error made on maximum shear forces is small, but the maximum shear force obtained by the model reduction without completions is higher, which makes this strategy accurate and conservative for the prediction of the maximum shear forces. Conversely, the distribution is poorly represented as shown by Fig 12 in accordance with the results of the semi-analytical approach 


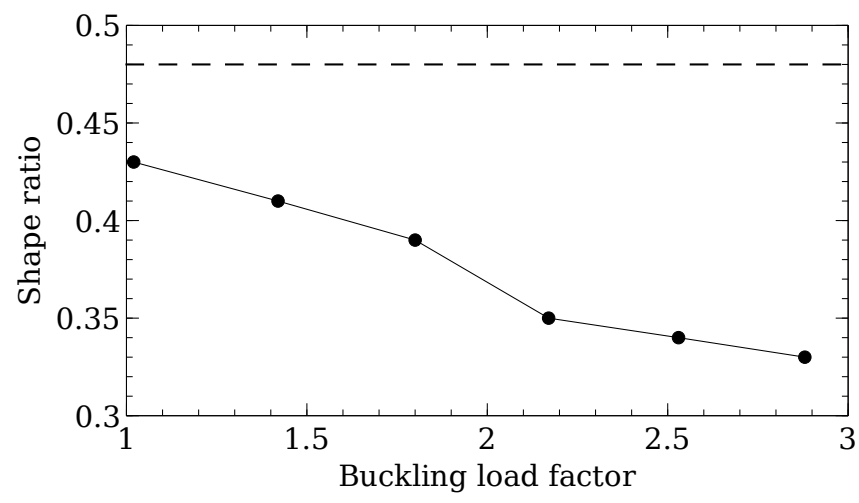

Figure 9: Evolution of the shape ratio of the displacement field over loading in terms of buckling load factor. Dashed line recalls the value of the shape ratio of the buckling mode displacement field.

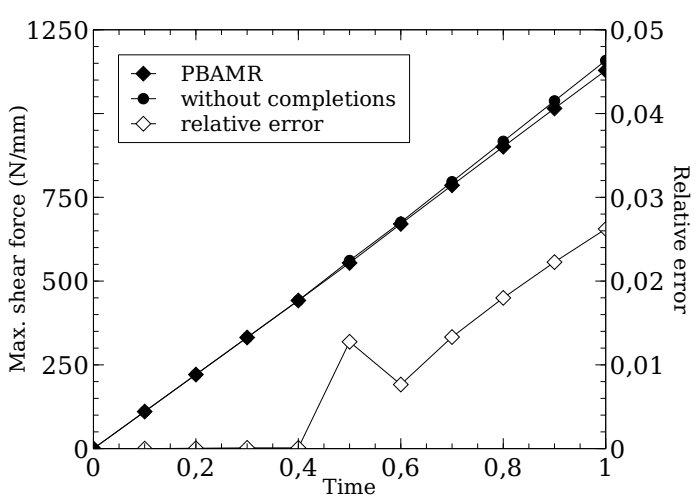

(a)

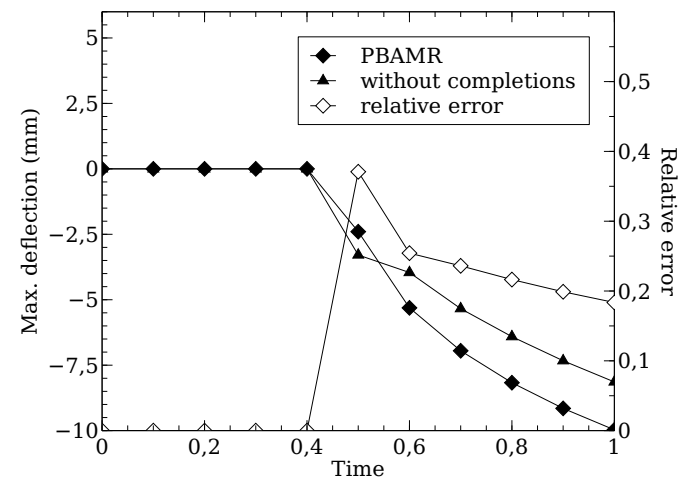

(b)

Figure 10: Comparison of the maximum shear forces (a) and the maximum deflections (b) obtained by the PBAMR strategy and model reduction without completions.

by Bisagni [18, and the maximum deflection is underestimated by more than $18 \%$. The weaknesses of the model reduction without completions are the results of an uncontrolled approximation error (i.e. normalized residual forces), whose evolution over increments is reported in Fig 11 and compared to those of full NewtonRaphson procedure and PBMAR strategy.

\section{Parametric study}

Even though the computed additional basis vectors allow the post-buckling displacement to be approximated with a given accuracy, the influence of the parameters on the efficiency of the procedure has to be identified. The aforementioned completion parameter $k$ and the convergence parameter $\epsilon_{N e w}$ were the only two parameters of the proposed PBAMR strategy. Fifteen numerical tests were carried out with the same FEM and loading. In Fig 13 the number of completions is presented versus the completion parameter $k$ in the range between $10^{-4}$ and $5 \cdot 10^{-1}$. The same test was performed for three values of the convergence 


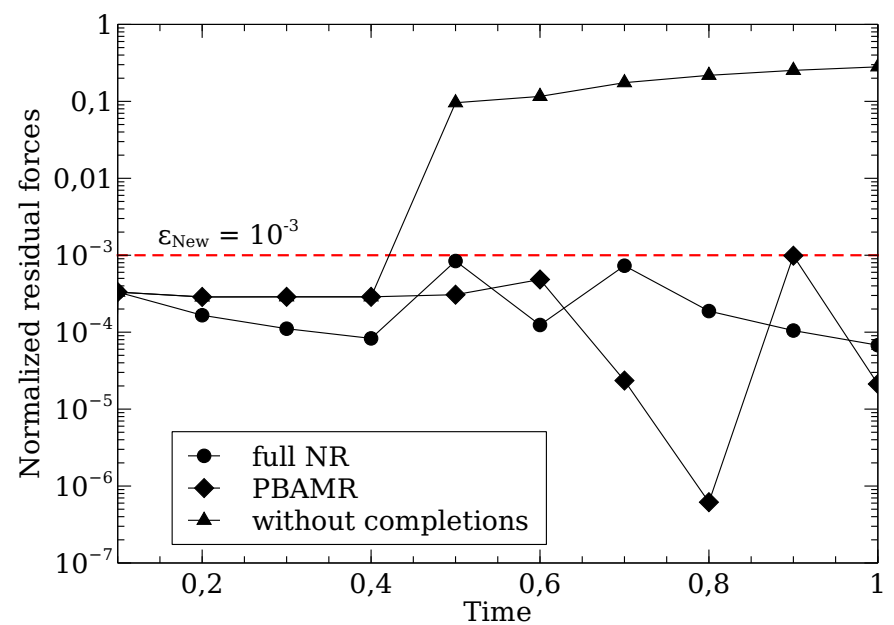

Figure 11: Comparison of approximation errors obtained by full Newton-Raphson (full NR), PBAMR and model reduction without completions. The convergence parameter $\epsilon_{N e w}$ is recalled by the dashed-red line and fixes the accuracy of full NewtonRaphson and PBAMR procedures only.

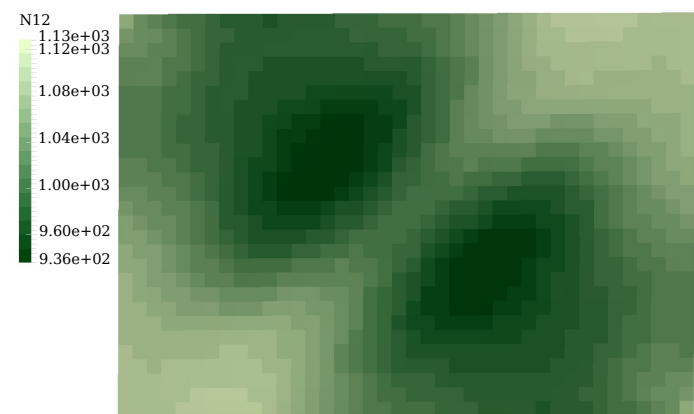

(a)

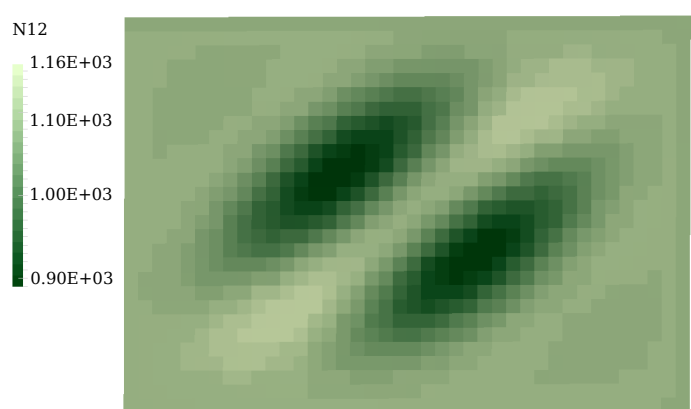

(b)

Figure 12: Comparison of the distribution of the shear forces obtained by the PBAMR strategy (a) and by the model reduction without completions (b). 
parameter.

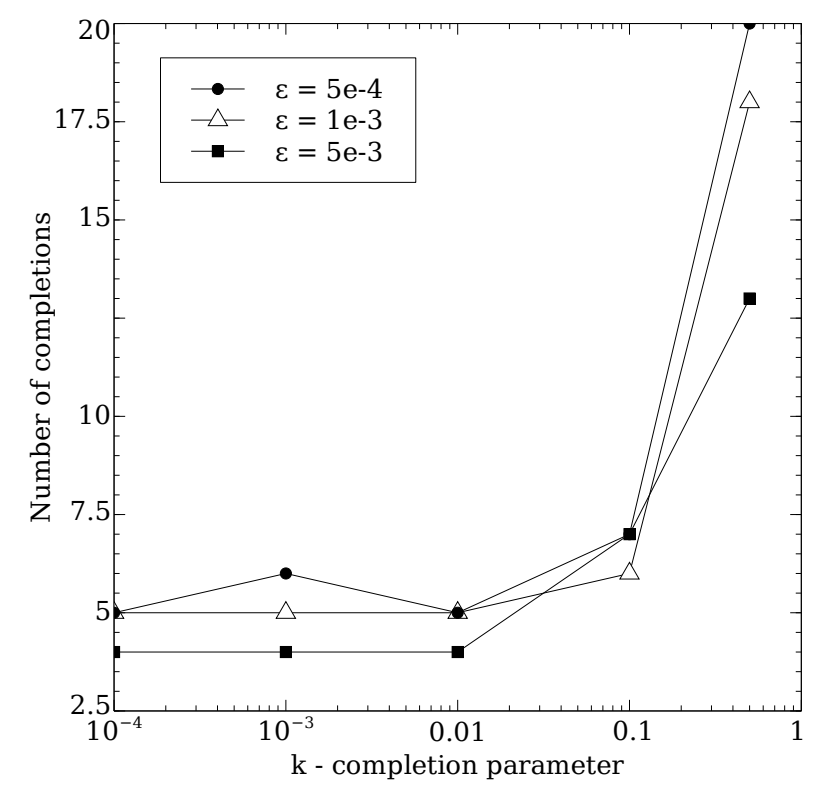

Figure 13: Influence of completion parameter $k$ and convergence parameter $\epsilon_{N e w}$ on the number of completions

Although a minimum number of completions is highlighted for values of $k$ under $10^{-2}$, the number of iterations, which is reported in Fig 14 , is a decreasing function of $k$ in this range.

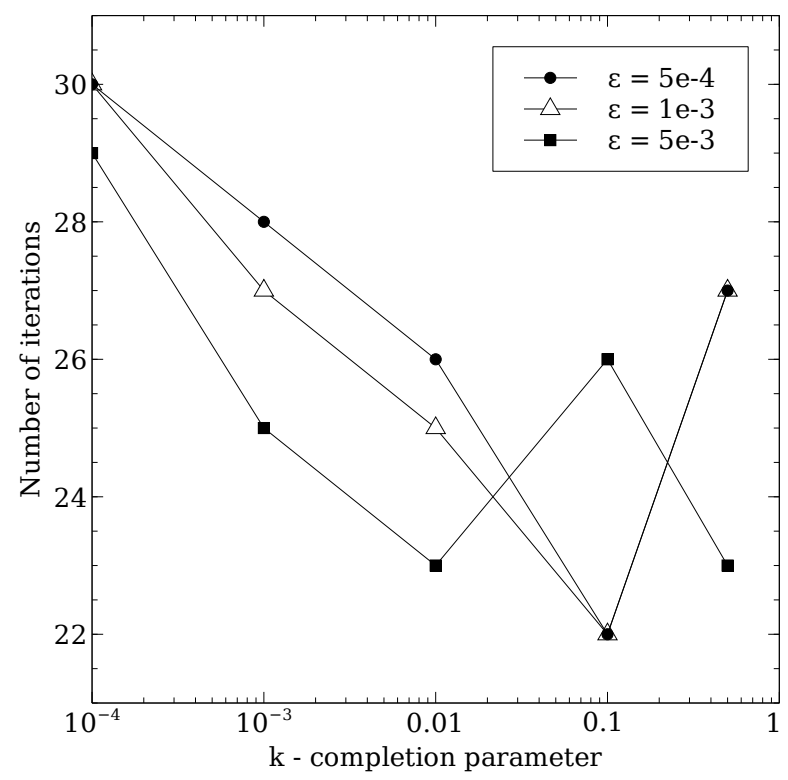

Figure 14: Influence of completion parameter $k$ and convergence parameter $\epsilon_{N e w}$ on the number of iterations

Kerfriden et al. [1] observed the same behaviour, which was explained by the following key points: 
- By definition of the criterion, a completion is more likely to be triggered when a large value of $k$ is set. $e_{C}$ being the projection of $e$ into a reduced subspace, a completion is performed at each iteration from $k=1$ or more (see Fig, 1 ).

- Since a minimum number of completions is required to reach the given accuracy of the approximation, there is a threshold at which decreasing the value of $k$ does nothing but delay the triggering of the completion (increasing the number of iterations).

From the present parametric study, although it could depend on the problem, the influence of the parameters of the strategy has been partially described and an optimal value for the completion parameter $k$ is proposed in this light. Among the values of $k$ tested, $10^{-2}$ is the one that achieves both the minimum number of completions and a reasonable number of iterations (23 iterations versus 21 full Newton iterations with $\epsilon_{\text {New }}=5.10^{-3}$ ). The convergence parameter, which is fixed by the user, is kept equal to $5.10^{-3}$ in the following developments.

The chosen parameter values are confronted with the mesh-refinement of the FEM in Tab3

\begin{tabular}{l|ccc}
\hline Mesh refinement (d.o.f.) & 885 & 3865 & 8945 \\
\hline Completions & 4 & 4 & 4 \\
\hline Iterations & 20 & 23 & 22 \\
\hline
\end{tabular}

Table 3: Influence of the mesh refinement on the number of completions and iterations $\left(k=10^{-2}, \epsilon_{N e w}=5.10^{-3}\right)$

It is interesting to note that the mesh-refinement has almost no influence.

In a more representative application of the industrial problem, the next section tries to validate the observed behaviour and to demonstrate the computational capabilities of the strategy, which have not been assessed yet.

\subsection{Computational performance: application to a stiffened panel}

The PBAMR strategy is applied to the finite element simulation of the stiffened panel presented in Fig 15 The stiffening is imposed by boundary conditions on the out-of-plane and rotation degrees of freedom. The panel is subjected to a shear loading that is currently observed in aeronautical applications such as the one prescribed by the deformable square test [2]. The displacement is imposed on the edges as follows to reproduce a rigid articulated frame:

- $u(x, y)=0.018 \times y$

- $v(x, y)=0.018 \times x$

- $w(x, y)=\theta_{1}(x, y)=\theta_{2}(x, y)=0$ 
The force $F$, which is drawn in Fig, 15 , is the equivalent reaction force on two diagonally opposite corners of the test frame. Since stiffeners are non-uniformly distributed on the panel, shear buckling starts in the widest sub-panel between stiffeners.

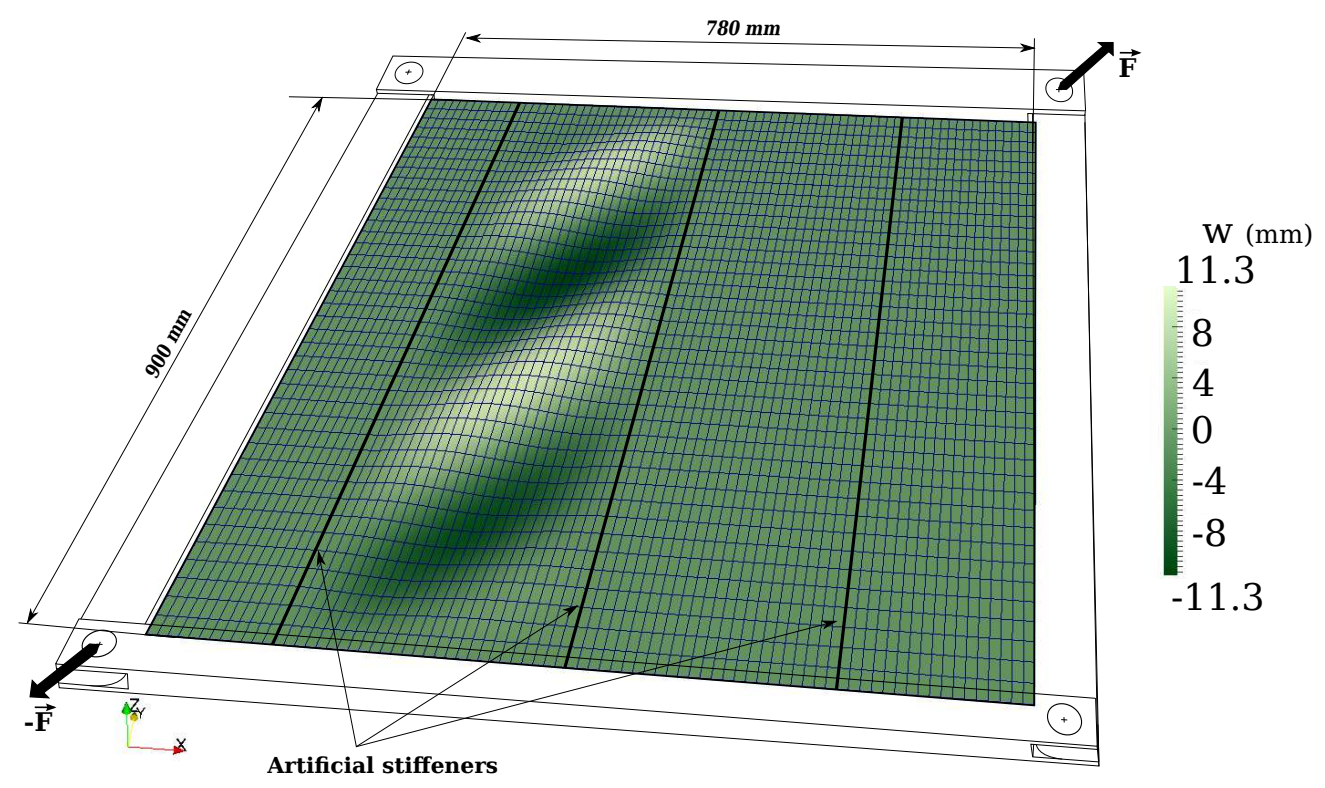

Figure 15: Application to an artificially stiffened panel. Local post-buckling under pure shear loading.

The loading reaches 2.75 times the first critical buckling load, whose value is $F_{c r}=3320 \mathrm{kN}$ in terms of equivalent reaction force. The displacement is imposed on the edge in 10 increments, as follows:

- The first time increment, that is used to build the initial reduced basis, is solved by a linear analysis. $\underline{U}_{\mid t_{0}}$ and the first buckling mode are thus computed to form the initial reduced basis.

- In the pre-buckling range of loading, the time increments are solved with the PBAMR strategy. The load reaches $90 \%$ of the first critical buckling load computed previously, in order to remain in the pre-buckling stage. Due to linear behaviour of the present structure before buckling, this load level is reached in 1 increment and no completions are expected.

- In the range of post-buckling, the next 8 time increments are also solved with the PBAMR strategy.

The present study was conducted over three mesh refinements of the same model in order to highlight some scalability properties of the proposed strategy. The maximum size of the model is 16774 degrees of freedom (d.o.f.).

First, the behaviour of the strategy is presented in Fig 16 . The number of completions is observed throughout the evolution of the size of the reduced basis over iterations. It is shown that the completions 
are regularly distributed over post-buckling increments. More precisely, less than one on the fly completion is performed per time increment in the post-buckling range of loading.

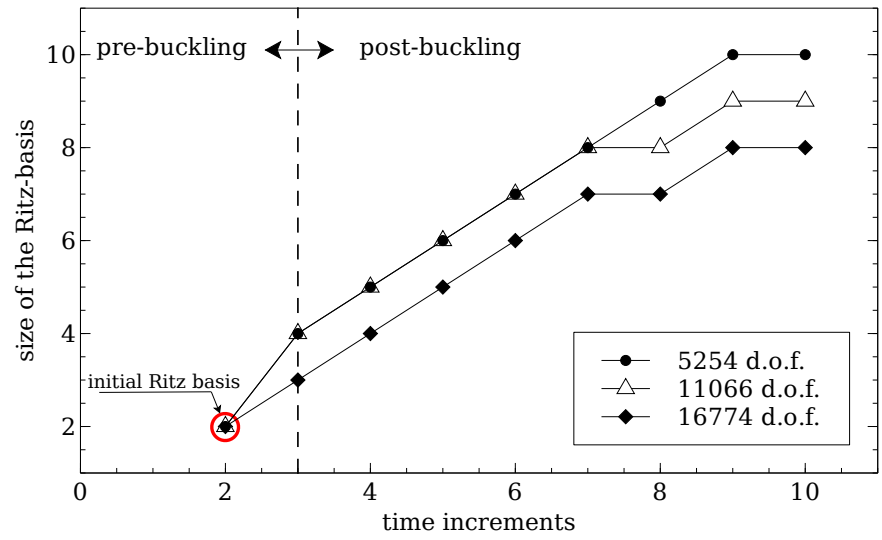

Figure 16: Behaviour of the strategy: evolution of the size of the reduced basis over increments for different mesh-refinements.

The number of iterations is reported in Fig $17 \mathrm{~b}$, in addition to the computation times of the tangent systems solving method Fig $17 \mathrm{a}$, which was cumulated over iterations. The computation times of the assembly operations are very dependent on the programming and are not reported here. Conversely, the solving of linear systems depends on standard Python numerical libraries, which makes results less dependant on code.

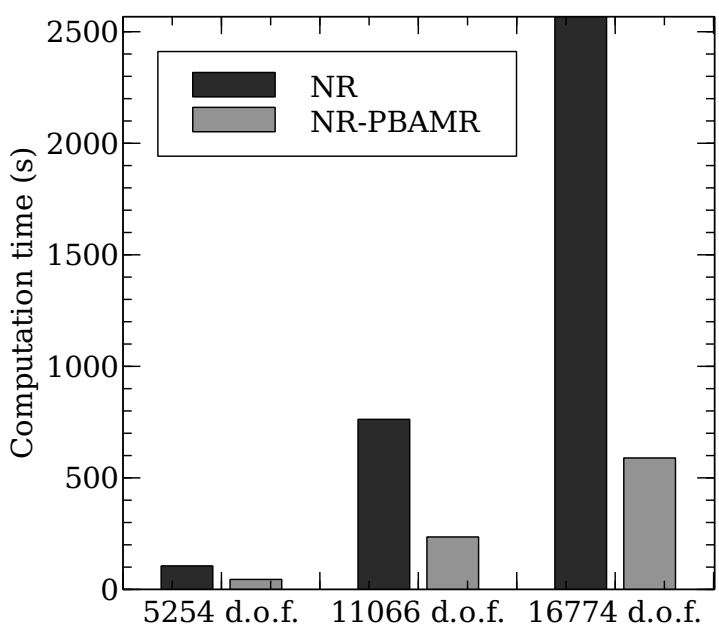

(a)

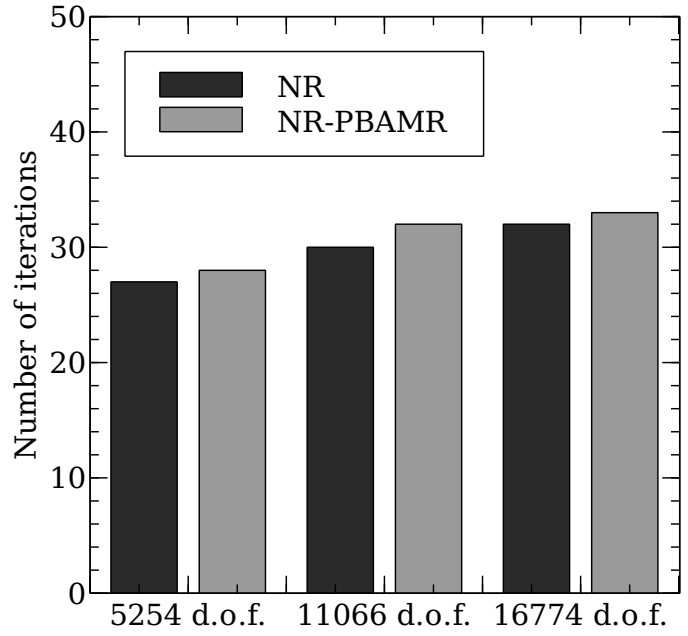

(b)

Figure 17: Computational performance of the PBAMR strategy for different mesh refinements. (a) Computation time and (b) number of iterations as compared with the computation time of the full Newton-Raphson procedure (NR).

The computation time reduction due to the PBAMR strategy was calculated and is presented in Fig 18 It can be observed that the efficiency increases with the size of the FEM. This is consistent with the evolution 
of the ratio between a reduced and a full Newton iteration. Even though the relative computational cost of an on the fly completion (enhanced iteration) also decreases, it is slightly higher that of a full iteration, which probably results from poor conditioning of the enhanced problem in the case of post-buckling of plates. Further developments are required on this particular point.

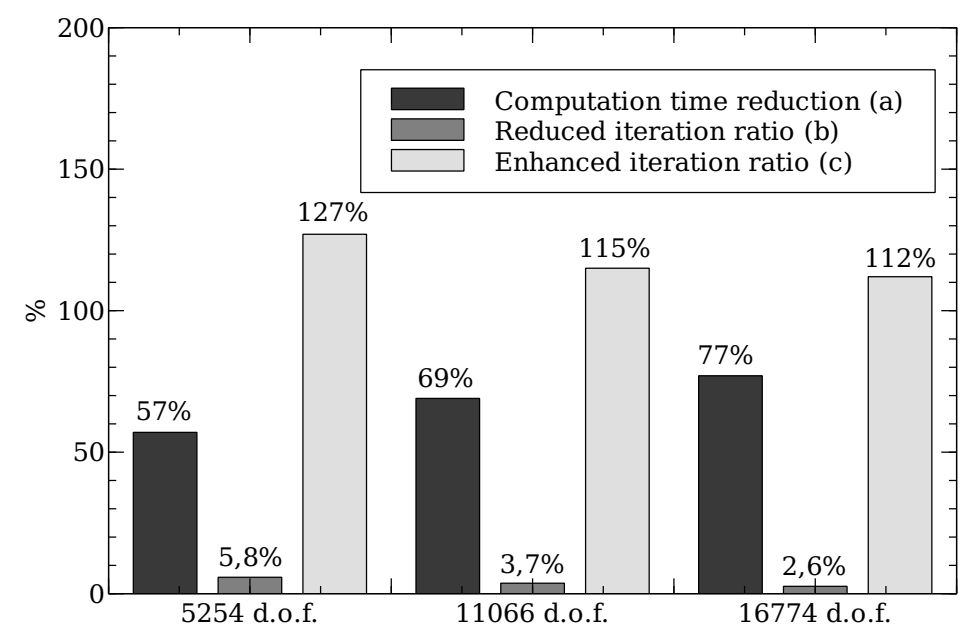

Figure 18: (a) Computation time reduction due to the PBAMR strategy in comparison with a full Newton-Raphson procedure. (b) Ratio between a reduced iteration and a full Newton iteration. (c) Ratio between an enhanced iteration (on the fly completion) and a full Newton iteration

\section{Conclusion and Perspectives}

In the framework of model reduction techniques, a strategy has been developed that reduces the computational cost of post-buckling simulations. This approach makes the most of an on the fly adaptive procedure and little knowledge on post-buckling phenomenon. More precisely, as stated in the semi-analytical methods, the post-buckling equilibrium state of structures is taken to be a combination of the pre-buckling state (known) and a variation, which is decomposed into a buckling mode component and a higher order variation arising from an automatic completion procedure. This results in a fast algorithm for solving post-buckling problems, which does not require expensive pre-calculations and is positioned both as an alternative to POD-based model reduction and as a way to build POD snapshots for post-buckling analysis at lower cost.

In this paper, the relevance of this approach has been demonstrated in the case of post-buckling analysis of plates. In spite of the limits of the home-made finite element research code, insight was gained into the computational performances of the strategy for an in-plane load reaching more than twice the buckling load and a finite element model with up to $1.6 \times 10^{4}$ degrees of freedom. The computation times were reduced by $77 \%$ and are expected to be even further reduced as model size increases.

According to the application case, the scope of the post-buckling adaptive model reduction strategy should include the post-buckling analysis of common stiffened structures. Few on the fly completions can be 
expected as long as the assumption of a small higher order variation is verified. Otherwise, the computation times should increase with the number of completions.

Although post-buckling bifurcations (buckling mode switching) are commonly observed in the targeted applications, they are not currently handled by the strategy. As mentioned in this paper, further work should focus on bifurcation branch detection and reduced basis updating. The localization of post-buckling of some stiffened structures, as observed in the proposed application, would also require a specific treatment. The cost of the completion could be reduced by localization of the completion in the buckled area. Thus, our research is now focusing on the coupling of the post-buckling adaptive model reduction strategy with domain decomposition methods [8, 29] in order to limit the costly completions to the substructures subjected to buckling.

\section{Acknowledgements}

The authors wish to acknowledge EADS Innovation Works for support and funding. This work was granted access to the HPC resources of CALMIP under allocation 2012-P12152.

\section{References}

[1] J. Bertolini, B. Castanié, J.-J. Barrau, J.-P. Navarro, Multi-level experimental and numerical analysis of composite stiffener debonding. part 1: Non-specific specimen level, Composite Structures 90 (4) (2009) 381 - 391. doi:D0I:10.1016/j. compstruct.2009.04.001

[2] J. Bertolini, B. Castanié, J.-J. Barrau, J.-P. Navarro, C. Petiot, Multi-level experimental and numerical analysis of composite stiffener debonding. part 2: Element and panel level, Composite Structures 90 (4) (2009) $392-403$. doi:DOI: 10.1016/j.compstruct.2009.04.002

[3] R. Vescovini, C. Dávila, C. Bisagni, Failure analysis of composite multi-stringer panels using simplified models, Composites Part B: Engineering 45 (1) (2013) 939-951. doi:10.1016/j.compositesb.2012.07.030

[4] C. Bisagni, L. Lanzi, Post-buckling optimisation of composite stiffened panels using neural networks, Composite Structures 58 (2) (2002) 237-247. doi:10.1016/S0263-8223(02)00053-3

[5] L. Lanzi, V. Giavotto, Post-buckling optimization of composite stiffened panels: Computations and experiments, Composite Structures 73 (2) (2006) 208-220. doi:10.1016/j.compstruct.2005.11.047

[6] E. Byklum, E. Steen, J. Amdahl, A semi-analytical model for global buckling and postbuckling analysis of stiffened panels, Thin Walled Structures 42 (2003) 701-717.

[7] R. Vescovini, C. Bisagni, Single-mode solution for post-buckling analysis of composite panels with elastic restraints loaded in compression, Composites Part B: Engineering 43 (3) (2012) 1258-1274. doi:10.1016/j.compositesb.2011.08.029

[8] P. Cresta, O. Allix, C. Rey, S. Guinard, Nonlinear localization strategies for domain decomposition methods: Application to post-buckling analyses, Computer Methods in Applied Mechanics and Engineering 196 (8) (2007) 1436-1446. doi: $10.1016 / \mathrm{j} . \mathrm{cma} .2006 .03 .013$

[9] J.-C. Passieux, P. Ladevèze, D. Néron, A scalable time-space multiscale domain decomposition method: adaptive time scale separation, Comput. Mech. 46 (4) (2010) 621-633.

[10] C. Farhat, K. Pierson, M. Lesoinne, The second generation FETI methods and their application to the parallel solution of large-scale linear and geometrically non-linear structural analysis problems, Computer Methods in Applied Mechanics and Engineering 184 (2-4) (2000) 333-374. doi:10.1016/S0045-7825(99)00234-0

[11] P. Kerfriden, P. Gosselet, S. Adhikari, S.-P.-A. Bordas, Bridging proper orthogonal decomposition methods and augmented Newton-Krylov algorithms: An adaptive model order reduction for highly nonlinear mechanical problems, Computer Methods in Applied Mechanics and Engineering 200 (5-8) (2011) 850-866. doi:10.1016/j.cma.2010.10.009

[12] R.-R. Craig, M.-C.-C. Bampton, Coupling of substructures for dynamic analyses, AIAA JOURNAL Vol. 6 (1968) pages $1313-1319$.

[13] A.-K. Noor, J.-M. Peters, Reduced basis technique for nonlinear analysis of structures, AIAA Journal 18 (4) (1980) 455-462. doi:10.2514/3.50778

[14] D. Ryckelynck, D. Missoum Benziane, Multi-level a priori Hyper-Reduction of mechanical models involving internal variables, Computer Methods in Applied Mechanics and Engineering 199 (17-20) (2010) 1134-1142. doi:10.1016/j.cma. 2009.12.003 
[15] A. Kling, R. Degenhardt, R. Zimmermann, A hybrid subspace analysis procedure for non-linear postbuckling calculation, Composite Structures 73 (2006) 162-170.

[16] S.-P. Timošenko, J.-M. Gere, Theory of elastic stability, 2nd Edition, Engineering societies monographs, McGraw-Hill Kogakusha, New York Toronto London Tokyo, 1963.

[17] W.-T. Koiter, Buckling and post-buckling behaviour of a cylindrical panel under axial compression, Tech. Rep. NLL-TR S.476, National Aerospace Laboratory NLR (May 1956).

[18] C. Bisagni, R. Vescovini, Analytical formulation for local buckling and post-buckling analysis of stiffened laminated panels, Thin-Walled Structures 47 (3) (2009) 318-334. doi:10.1016/j.tws.2008.07.006

[19] W.-T. Koiter, On the stability of static equilibrium, Ph.D. thesis, Polytechnic Institue Delft (1945).

[20] M.-A. Crisfield, Non-linear Finite Element Analysis of Solids and Structures, Wiley, 1991.

[21] B.-O. Almroth, F.-A. Brogan, P. Stern, Automatic choice of global shape functions in structural analysis, AIAA Journal 16 (5) (1978) 525-528. doi:10.2514/3.7539

[22] C.-L. Chang, J.-J. Engblom, Reduced basis alternatives to the solution of nonlinear dynamical systems, Journal of Aircraft 29 (5) (1992) 760-767. doi:10.2514/3.46243

[23] D. Nagy, Modal representation of geometrically nonlinear behavior by the finite element method, Computers \& Structures 10 (4) (1979) 683-688. doi:10.1016/0045-7949(79)90012-9

[24] A.-K. Noor, C.-M. Andersen, J.-M. Peters, Reduced basis technique for collapse analysis of shells, AIAA Journal 19 (3) (1981) 393-397. doi:10.2514/3.7776

[25] K. Willcox, J. Peraire, Balanced model reduction via the proper orthogonal de- composition, AIAA 40 (2002) $2323-2330$.

[26] B. Falzon, D. Hitchings, Capturing mode-switching in postbuckling composite panels using a modified explicit procedure, Composite Structures 60 (4) (2003) 447-453. doi:10.1016/S0263-8223(03)00019-9

[27] S. Baguet, B. Cochelin, Determination of branches of limit points by an asymptotic numerical method, in: Proceedings ECCOMAS 2000, Barcelona, Espagne, 2000, p. 16pp.

[28] G. Van Rossum, F. Drake, Python Reference Manual, PythonLabs Edition, Virginia, USA, 2001, available at http://www.python.org.

[29] P. Kerfriden, O. Goury, T. Rabczuk, S.-P.-A. Bordas, A partitioned model order reduction approach to rationalise computational expenses in nonlinear fracture mechanics, Computer Methods in Applied Mechanics and Engineering doi:10.1016/j.cma.2012.12.004 